\title{
Using Persuasive Recommendations in Wellness Applications based upon User Activities
}

\author{
Hamid Mukhtar \\ Department of Computer Science, College of CIT Taif \\ University, Taif, 21974, Kingdom of Saudi Arabia
}

\begin{abstract}
Recently, a large number of mobile wellness applications have emerged for assisting users in self-monitoring of daily food intake and physical activities. While such applications are in abundance, many research surveys have found that the users soon give them up after giving some initial try. This article presents our application for healthcare self-management that monitors users' activities but -unlike the existing applicationsit focuses on keeping the users engaged for self-management. The distinguishing feature of our application is that it uses persuasive mechanisms to help users adopt healthy behavior. For this purpose, users' various activities are monitored and then they are persuaded using different persuasion strategies that are adaptive and are according to their behavior.
\end{abstract}

For each user, a behavior model is created that is based on Fogg's behavior model but, in addition, it also holds within it user preferences, and user health profile. The behavior model is then used to create a persuasion profile of the user that allows us to propose personalized suggestions targeted to overcome his lacking behavior. We also describe a case study that describes the actual application.

Keywords-user goal; modeling; user feedback; context; preferences

\section{INTRODUCTION}

To promote a behavioral change can be a challenge for most people as changing a lifestyle habit often means trying something new and different from the ordinary, and this has proven difficult. Karapanos [1] argues that contrary to the common belief that behavior change is the result of deep knowledge about one's own behaviors, they found that people rarely look back at their past performance data and may not have deep knowledge about their own behaviors. Rather, a glance of a brief, 5-second session make them knowledgeable about their behavior where users check how much they have walked so far without any further interaction.

As the healthcare costs have risen significantly, much of the recent healthcare research focuses on self-management of health. Systems have been developed that monitor users for their health-related behavior and make informed decisions to help them in attaining better health. Future environments are envisioned to be populated with such computational technologies that adapt to meet the needs of individual users. However, such technologies will be effective only if the users feel comfortable in adopting them. Unfortunately, while the existing solutions for healthcare self-management focus on the improvement of data acquisition and analysis methods, they have not achieved the desired outcome because of the resistance by the users to adopt them.

There are several reasons for them. First, as human beings, we are not interested to perform a task unless we associate it with some sort of outcome that is beneficial. Thus, it is unrealistic to expect that users will use some system without knowing or receiving any benefits from the system. That is why, when designing applications and systems for healthcare self-management, we should take extra measures and consider additional requirements as well. Second, usually such systems require the users to wear or carry-on additional accessories, usually electronic equipment, to keep track of their health. But people usually feel about them as an overhead that should be avoided. Third, self-management requires the user to be determined and undertake certain activities which may have compromising effects on their personal life-style. Since nobody likes such changes, people resist to adopt such systems which change the normal status quo of the users.

To address such issues, researchers have suggested the use of technology for persuading people for changing their behavior. Persuasive technology is defined as, "any interactive computing system designed to change people's attitudes or behaviors" [2]. Two of the most widely used persuasive technologies are mobile devices and websites. Mobile devices have added advantage for supporting behavior change because they are always in the ON state can intervene at the right time, in the right context, in a convenient way [2]. Moreover, virtually everyone has got a mobile phone so they are suitable for the development of persuasive applications. That is why most of the recent research work focuses on mobile phone based health intervention systems and applications.

However, everyone cannot be persuaded using the same approach. Different people are persuaded differently for different reasons related to their behavior, attitude, lifestyle, social setup, etc. For example, let's consider the health-related goal of Going for a 30 minute walk daily early morning. Although the goal is very clear and it should be achievable. However, most of the people fail to do so. Some people simply do not have the motivation to wake up early from the bed. Others may be motivated and would wake up early but they may not find the time for a walk. Similarly, some people will be both motivated and have time but they simply forget about it. For this purpose, various persuasion strategies can be used to persuade users and help them in changing their behavior. Each user can be persuaded by different strategies at different times. Previously, we developed a mobile phone application called Sedentaware whose purpose was to promote activeness in adults by monitoring their physical activities and providing them with appropriate feedback to adjust their behavior accordingly [3]. To make the application engaging for the users, we used several design techniques that would 
persuade the users to take preventive actions. The current work addresses some limitations of that work as well as proposes some enhancements to it.

One of the foremost motivating aspects used in our app is that of goal setting whereby the user is empowered to define their own means for achieving the desired level of fitness. In our case, the goal is set in terms of number of minutes of daily walk. Users feel more engaged when they have the ability to manage set their goals. Moreover, from persuasion point of view, this also means they abide by keeping to their goal more often. In case the users do not follow the goal, the persuasive messages try to get them back on track. As found by Karapanos [1], individuals who have the intention but not yet the means (i.e. motivation) to change, had an adoption rate of $56 \%$, whereas individuals in pre-contemplation, action or maintenance stages had an adoption rate of only $20 \%$.

In this article, we extend on our previous work and present our approach for healthcare self-management considering the above mentioned aspects. Our focus is more towards developing an intelligent system for recommending the users rather than solely following other users or doctors as done by various existing systems. As discussed in [3], users are monitored for activities using the sensors on their mobile phones and recommendations are provided according to their profile, health level, and preferences. In the current approach, the objective is to describe how to persuade users for behavior change using the appropriate strategies designed for this purpose. The persuasion strategies have been designed using a formal behavior model.

In addition to monitoring the users, the application also provides analysis using a rule-based system. On the basis of analysis outcomes, the user is suggested activities according to their past behavior and their current preferences. The activities are then assessed and compared with the user's health-related variables. In case of any conflict, a precisely defined goal is set for the user in the form of a target behavior to be adopted. Thereafter, the user is monitored for the new goal.

The remainder of the article is organized in the following manner. In the next section, we describe related work and advances in healthcare self-management as well as use of persuasive technology for that purpose. Section III explains the steps carried out in our design based upon the guidelines set forth by BJ Fogg. In Section IV, we explain the underlying model behind our approach and the developed mobile application on top of it. Section V, we explain how different persuasion strategies can be applied for behavior change based on Fogg's behavior model. Finally, in Section VI we describe the prototype implementation of our application considering diabetes self-management as a case study. We then conclude this article with a brief discussion of future work in Section VII.

\section{Related Research in Persuasive HealthCARE}

Mobile apps have been one of the most adapted mechanism persuasion, whether in healthcare, education, e-government, politics, etc. It is mainly because of the added value and benefit of mobile phones' ability to adapt day-to-day interactions based on previous usage patterns [4]. Moreover, mobile service benefits include extensive data capturing throughout the day to enable more participant empowerment, improved data quality for the health management process, improved feedback options based on improved data in different types of visualizations suited for the type of data, stimulating the ownership of their health by the users, more health data integration at the level of the person involved, etc. [5].

Persuasive technologies have been used in healthcare domain for: motivating healthier behavior using smartphones [6], motivating elderly individuals to walk [7] encouraging social and physical activities [8], self-management of chronic diseases such as diabetes, stroke, Alzheimers, obesity, etc. [9], promoting health education in children [10], and for changing existing social beliefs and health practices of rural women in a positive manner [11].

More relevant to our work is the UbiFit Garden system that was designed to encourage regular physical activity using persuasion [12]. The system mainly comprises of wearable sensors for detecting and tracking people's physical activities and displaying them through an aesthetic image in the form of a flower garden. When the sensors detect that user has performed some physical activity, the appearance of the garden improves by adding new elements, such as butterflies, flowers and plants. If no physical activity is detected from the user, the flowers in the garden perish slowly. This induces a sense of motivation in the users and they continue with their physical activities.

The Chinese Aged Diabetes Assistant (CADA) project $^{1}$ is using mobile device for healthcare management to enable multiple forms of input to accommodate users with very basic computer skills. It supports a range of self-management and educational activities needed for self-care. The data obtained can be readily shared with physicians given the existing infrastructure. To help users in adopting the system, this project is using the users' own cultural (Chinese) icons for eliciting and gathering user requirements and goals and also for the sake of education.

Lin et al. [13] have developed a Web 2.0 based diabetes support system with care provider perspectives. The system supports care managers in a health service center to conduct patient management through collecting patients daily physiological information, sharing care information, and maintaining patient-provider relationships. However, there is no support for other media including mobile phones. Moreover, it does neither consider patients' perspective nor it outlines how the social capabilities of Web 2.0 can be utilized to support patients.

After looking at all these efforts of assisting users for healthcare self-management, we find focus on monitoring the user's data and then giving feedback to them. A user is advised in some traditional way and then it is left to them how to bring in these changes for self-improvement. In our opinion, any system designed for wellness of users should be able to identify the lacking aspects and then it should include not only assistance but help users in motivating in that particular aspect.

Tatara et al. [14] conducted a survey regarding feasibility of using mobile phone based terminal for acceptability or effectiveness of self-management of diabetes. The finding was that acceptability factors depend on variables like motivation in

${ }^{1}$ http://www.cadaproject.com/ 
self-management, long-term adherence, relationship between patients, and long term application use among others. The research concludes that various factors such as lifestyles, skills, and cognitive abilities of patients need to be considered when using mobile-based healthcare applications.

Based on the analysis of above mentioned limitations and problems in the existing solutions, we now describe our application in detail.

\section{DESIGN RATIONALE}

B.J. Fogg proposed eight steps to outline a path to follow in designing persuasive technologies [15]. Considering these 8 steps as guidelines, we explain here our design methodology.

Step 1: Choose a simple behavior to target for change: In our work we have chosen the exercise habit (particularly walk for a specific duration) as our behavior target.

Step 2: Choose a receptive audience: During our research phase, we preferred to rely on the research team consisting of two Master students (aged 22 and 23), a PhD researcher (aged 29) and the supervisor (aged 54). Once a working prototype was developed, we chose three more participants (ages 24, 27, and 56) to test our application. Two of these were females while remaining five were male participants.

Step 3: Find what is preventing the audience from performing the target behavior: As explained later, it was about identification of motivation, ability and trigger factors that we designed for. The detail is given in later sections.

Step 4: Choose a familiar technology channel: We chose mobile phone technology channel.

Step 5: Find relevant examples of persuasive technology: There is quite a lot of work considered as examples from which we learnt. Some of them have been described in the related work.

Step 6: Imitate successful examples: As identified in the related work, we have incorporated different aspects of our own work as well as some from the notable peer-reviewed work of others.

Step 7: Test and iterate quickly: The application was testing constantly during development phase and issues were resolved on regular basis. The prototype was then tested on additional users.

Step 8: Expand on success: The last step has yet to be carried out as we have not yet done extensive experiments on a large group of users.

Having outlined our steps, we now go into the technical details of our scientific approach.

\section{APPLICATION ARCHITECTURE FOR PERSUASIVE HEALTHCARE RECOMMENDATIONS}

Persuasive technology is centered on the concept that persuasion can be carried out by machines instead of human with the objective to induce some change in their behavior. Persuasion is a complicated process and cannot be carried out using an automated set of certain steps thanks to the complex behavior of human being. One of the important findings from studies on extensive lifestyle interventions is that to maximize health benefits, large lifestyle changes are needed. The changes cannot be achieved unless they are being monitored by some entity. In addition, the monitoring must also contain aspect of assessment to determine the progress. That is why a good system should include both the aspects of monitoring and assessment.

\section{A. The Monitoring-Assessment Model}

The foundation of our persuasive healthcare application is the monitoring-assessment model that has seven stages as shown in figure 1. Its different components have been identified in the conceptual framework of the application. Each one roughly correspond to some stage of the model as depicted in figure 2. Each of the seven stages is described next in some detail.

1) User Profiling: This is the preparatory stage and is used to get user information for creating user profile. This is only a onetime process required. User profile is any predefined or existing information related to the user. As shown in figure 2, profiling comprises preferences of the user, their health profile and building the user model from both of them collectively.

The health profile contains basic information about their demographics (name, age, gender, etc.) as well as medical history, chronic conditions, disabilities, and other health-related parameters. Preferences include user's choices about exercise type and routine, their diet, and social interests that are used in the later stages. For profiling, we mainly rely on monitoring of user behavior using automatic means of data gathering by the mobile phones.

2) Target Behavior: Our objective is to overcome the weaker aspects of a user's behavior which are affecting his health by persuading them to perform some activities. In this regard, the system identifies a target behavior to be changed, which can be inferred by the user profile or as recommended by the experts. From user profile we can determine the lacking aspects (or weak points) of the user with respect to their activities or behavior, e.g., lack or need of exercise, irregularities in taking medicine or moderation in the intake of particular food; all these are some form of behavior that require an improvement and are candidates for target behavior.

a) Behavior Modeling: The user's behavior model allows understanding the user and in proposing them personalized recommendations and persuasion strategies for behavior change. Such a model is built using user's preferences and from their health profile (including history of diet, exercise and physical activities). In the literature, a number of behavior models and theories have been defined [16][17][18][19]. Of all these, Fogg's behavior model [18] has been primarily developed for persuasive behavior design and, being a generic model, we also adopted it for behavior change in our application.

BJ Fogg has identified three factors [18] that are necessary for invoking a behavior change. They are: motivation, ability and triggers. Our proposed behavior model uses these factors to devise the appropriate persuasion strategies for behavior change. 


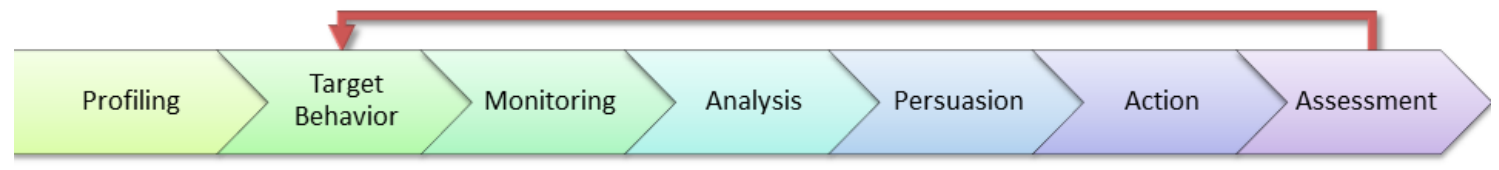

Fig. 1. The monitoring-assessment model

Existing literature differentiates between two types of motivation: extrinsic and intrinsic. Also, research suggests that it's the intrinsic (or internally driven) motivation that lasts longer and is helpful in bringing about a change in ones behavior. Although any external factor (e.g., mobile phones in our case) that persuades a user for motivation will be necessarily an extrinsic motivation factor, we believe that if a user observes even a small, possible change in behavior, they may be intrinsically motivated to promote and enforce the behavior. Thus, while we model the human behavior with respect to mobile phone technology, it will be ultimately part of once daily activities.

Keeping a user in the loop is an important aspect of the persuasion process. Many studies have indicated that participants fall back into less healthy or the previously held states once the intervention is over [20][21]. But it all starts with the monitoring of activities.

3) Acitivities Monitoring: The user's daily activities are observed to gather data related to physical activities, exercise, food consumption, and medication. The gathered data is used along with the information about the context of the user in the later stages of the model.

a) Context Gathering: Human behavior identification is a complex problem and consists of multiple factors. In addition to the state of the users such as posture and position of the body, there are a number of other factors outside the human body that are to be considered to infer the human behavior. To learn about the user behavior and to reason on them, we need to know the context in which an activity is performed. Context is any relevant information that help us in determining the type of activity, the state of the user and their surrounding environment at that time. Some of the context variables have been identified in figure 2 . These variables can be obtained from user's device.

To monitor user activities, we collect data related to the activities and behavior from the user on regular basis. We can monitor user for activities such as walk, stairs or cycling, still position and in vehicle (reader is referred to [3] for details on this aspect.) Although not implemented in our system, additional activities like eating, exercising, sleeping, and watching television can be carried out using previously developed approaches [22][23][24][25]; however, this discussion is beyond the scope of this article. Moreover, the users are able to enter information about their diet and medication into the application manually.

The identified activities relevant to our need are recorded in user's history and become part of the user's profile. It is used together with user's preferences and their behavior model and is used for persuasion.
4) Analysis: This stage is the intelligent processing part of our application. Here, the volume of data from monitoring stage together with various profile elements is analyzed using previously defined rules. The aggregator consolidates the data obtained from various sources (mobile sensors and user input) while the analyzer performs analysis on the gathered data and compares it with the available guidelines for validation. Finally, the assessor evaluates the data for inconsistencies. For analysis, we used rule-based inferencing. It is used to represent knowledge of a particular domain and deduce results on top of it. The knowledge base maintains the required knowledge for the particular domain and is populated from various clinical guidelines for healthcare such as found in both classical and modern findings in research [26][27][28][29]. If the knowledge base has enough data, rules can be constructed out of it and then the current working knowledge will be evaluated against it by chaining rules together until a conclusion is reached. A rule-based system makes the storage of a large amount of information easier, and coming up with the rules help in clarifying the logic used in the decision-making process. Here we are not concerned with the rule-based analysis and this will be described in a future article.

5) Persuasion: If it is found that the user's behavior is lacking in some of the aspects (e.g., diet, exercise, or medication), then they should be persuaded to improve on the lacking behavior. The user can be persuaded through various strategies for behavior change. These strategies have been adopted from the literature. Each strategy is relevant in a particular context. That is why we have defined rules that are used to match what is required with what is to be done in that context. Some of the strategies are discussed in Section V.

One of the important aspects of persuasion is to generate fast results to the users so the users can assess the difference, or no difference, or acting on the persuasion. This has to be achieved using frequent reminders and recommendations to the users [30].

6) Action: The purpose of a persuasion strategy is to induce some desirable, positive behavior change in the user. If the strategy is successful, it will influence the user who will take some measures or corrective actions. Such actions will contribute toward the self-management of healthcare. Both the actions and inactions of the users are recorded by the system (devices or sensors) as explained previously in the monitoring stage. In the future, we tend to consider the failure of a strategy when it does not influence the user. This will required a pipeline of strategies and adaptivity in the system with respect to different contexts.

a) End-User Adaptation: A user is persuaded to change behavior by applying an appropriate persuasion strategy that is designed for a particular action. An important part of our application is that persuasion strategies vary from one 


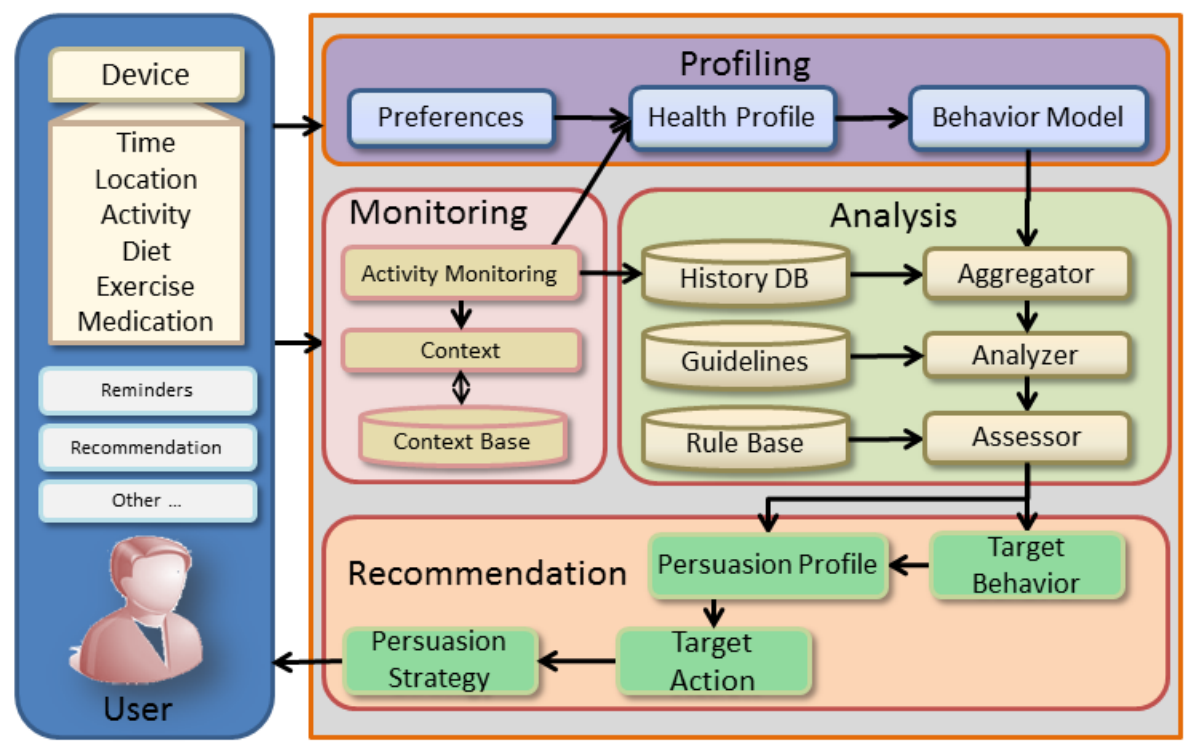

Fig. 2. The various components of mobile based persuasive application

context to another. For example, to persuade a user to exercise regularly, mobile devices will use reminders but to increase the intensity of activities, the strategy will be to show the user their achievement using physical activity data. Thus, the application interface with which the user interacts will be different in both cases. In other words, the user interface of the end application adapts itself according to the target user action. We will discuss example strategies in the case study in Section VI.

One aspect of end-user adaptation is to make the user interface fun and enjoyable. It should also support the users in the reconstruction of recent past actions either for comparison purposes, reflection, or for the satisfaction of the user.

7) Assessment: During assessment, a conclusion is drawn whether the analysis has been successfully applied through some persuasion strategies to materialize any behavior change in the user and, if it is, then any action was performed by the user as a result. This is done both quantitatively (yes/no) and qualitatively (how much time, amount, etc.) Based on the outcome of the assessment, either an improved target behavior is identified, such as more duratino for exercise, or the previously targeted behavior is strengthened by applying additional persuasion strategies.

An important aspect of assessment is that it should help the user build self-esteem and healthy attitudes and habits. In addition, it should also foster the attitude of care and love for oneself [31].

In the next section, we describe our persuasion strategies and how they can be used for behavior changes.

\section{Persuasion Strategies And Their Use in BEHAVIOR CHANGE}

A number of generic persuasion strategies have been described in the literature, e.g., in [32][33][34]. Similarly, various specific persuasion strategies have been designed by researchers and they have reported positive results in areas such as obesity, management of smoking cessation, asthma, stress and insomnia [35], directing users towards proper exercise behaviors [36], and healthier eating [37] among others. All of these approaches target behavioral changes in the end-users through some persuasion strategies. Before outlining how these strategies are used in our system under different contexts, let us briefly describe Fogg's behavior model, which forms the foundation of persuasive technology [2][18][17] as well as the basis for use of persuasion strategies in our application.

\section{A. Fogg's Behavior Model}

Fogg's behavior model [18] has three important factors or dimensions: motivation, ability and trigger. The model asserts that for a target behavior to happen, a person must have sufficient motivation, sufficient ability, and an effective trigger. All three factors must be present at the same instant for the behavior to occur.

a) Motivation: A person who has high motivation for performing the target behavior would be more likely to perform the target action. For a low-motivation person, one or more strategies pertinent to motivation should be used to increase the motivation. Users can be motivated by persuading them to do something for pleasure, to achieve some status or to expect some reward upon performing some activity.

b) Ability: A person having the required ability to perform a target behavior would be highly likely to perform the target action as compared to a person who is weak or lacks such an ability. To improve one's ability, one or more strategies relevant to ability should be used to enable the person. Examples of ability are: giving additional time to perform an activity, reducing the effort to do some activity, etc.

c) Trigger: A trigger is a signal, an event or its associated action that can be used to increase motivation or ability or both. In most cases, without an appropriate trigger, behavior will not occur even if both motivation and ability are 


\begin{tabular}{|l|l|l|l|}
\hline Strategy & Dimension & Meaning & Example Use \\
\hline Commitment & Motivation & $\begin{array}{l}\text { Once a user agrees for taking action, he will } \\
\text { commit to carry it out }\end{array}$ & Accepting a recommendation by the application \\
\hline Reward & Motivation & Offer the user reward on tasks accomplishment & Every action is logged and taken into account \\
\hline Effort & Motivation/Ability & $\begin{array}{l}\text { Make it easy for users to take actions } \\
\text { walk or exercise }\end{array}$ \\
\hline Achievement & Motivation & Keep the user updated about their progress & Provide daily, weekly, monthly statistics on how the user performs \\
\hline Personalization & Ability & $\begin{array}{l}\text { Provide the information that makes it easy to } \\
\text { understand and act }\end{array}$ & User can set his medication/exercise information \\
\hline Recommendation & Trigger/Motivation & Suggest a user an action to take & $\begin{array}{l}\text { End-of-day or end-of-week recommendation to compensate for } \\
\text { exercise deficiency }\end{array}$ \\
\hline Loss Aversion & Trigger & $\begin{array}{l}\text { Appropriate reminder if user misses to take an action (e.g., } \\
\text { medication) }\end{array}$ \\
\hline \multicolumn{2}{|l|}{ TABLE I. } & DIFFERENT PERSUASION STRATEGIES AND THEIR CONTEXT OF USE IN SEDENTAWARE APPLICATION FOR WELLNESS
\end{tabular}

high. We used reminders, alarms, and notifications as well as recommendations to trigger user action.

Table I shows a list of persuasion strategies, the dimension related to each strategy, their meanings and the context in which they can be applied. For example, the "effort" strategy can be applied to both ability and motivation of a person as it is useful to either increase the ability or the motivation levels of the user. On the other hand, the "reward" strategy will only increase the motivation of a person but not the ability. Using these three factors as guides, in the next few sections, we describe how various persuasion strategies can be used to design for behavior changes. It is worth noting that a single strategy can be applied in different contexts. For example, "reward" is equally applicable for rewarding the user on successful exercise but also on taking appropriate amount of diet and taking medicines regularly, etc.

The system can persuade the users in more than one ways for the same desirable target behavior change. Let's consider, once again, the example of the commitment strategy, which states that when a user commits to achieve a certain objecitve or goal, they are motivated to carry out the required activities. The application initiates commitment strategy by helping the user to set relevant goals. Similarly, the user is also persuaded through reminders and context-based suggestions so activities can be performed with reduced effort. In such cases, mobile phones come handy to remind the users about proposed recommended activities based on scheduled alarms or appropriate notifications. Similarly, they keep the users updated about their health status and generate notifications in case of divergence from the specified goal.

To propose strategies according to user's activity and history, the persuasion profile of the user is used in addition to considering their preferences and health data. We combine the persuasion profile with the context information and rules to determine the target behavior. User's previous actions are assessed and taken into account before suggesting a target behavior. This is depicted in figure 2 , where we translated a target behavior into a target action for the user. A persuasion strategy is then designed that adapts the user interface accordingly.

\section{B. The Role of Design in Persuasion}

According to Change et al., wearable technologies provided more awareness than motivation in physical activities with goal-setting and progress monitoring [38]. Thus, they are no sufficient to induce the required level of motivation that will let a user exercise more regularly. As concluded by the study, the use of wearable devices was not sufficient to rely on for regularity in physical activities. According to the study, the participants cited various reasons such as being too busy to exercise, finding exercise boring, poor self-management, fear of injury, lack of skills, and lack of encouragement, support, or companionship from family and friends [38].

\section{Diabetes Self-Management: A Case Study}

Diabetes is a chronic disease that is associated with a metabolic disorder in the human body. Managing diabetes is not an easy task as it needs serious attention by the user. Successful management of diabetes can have dramatic improvements in one's life as it relates to the overall user's life style and affects most of the user's activity. Healthy life style that includes regular physical activity and eating in moderation plays key role in slowing down diabetes related complications. Typically, diabetes has three main behavioral aspects that include diet, medication, and exercise [39]. For successful management of diabetes, all these aspects should be controlled simultaneously. This means taking help from trained healthcare professionals, coordinated care, user education and self-management training [27].

A user's complete and precise data regarding medication, diet, and exercise play an important role in managing their glucose level. This data is used by the experts to advise and suggest the users accordingly. However, since the amount of data recorded on daily basis is of different variety and huge in size, it is not feasible to rely on manual data maintenance mechanisms. This also makes it difficult for doctors to advise a user better.

Considering these complexities in diabetes selfmanagement, we were interested in it as a case study for our research approach. In this section, we explain how our proposed approach for user persuasion is applied practically. We then describe the implementation of the proposed system in the context of persuasion.

We have implemented a smartphone application for the Android platform. As part of the persuasion in the application design, the application has been created as a service that runs inside an Android widget. Being a widget means that the user does not need to explicitly start the application to use it nor the user needs to remember to use the application. The application always remains in active state on the home screen of the smartphone. It starts automatically with the Android boot process and is brought onto the screen whenever the user unlocks the phone for any activity. Being a service means that the application continuously monitors user activities and 


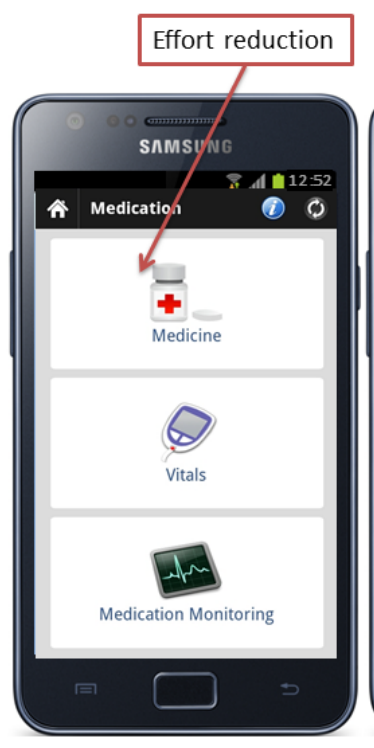

a)

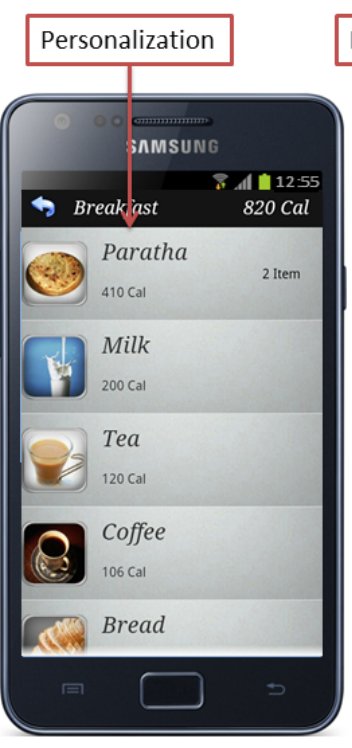

b)

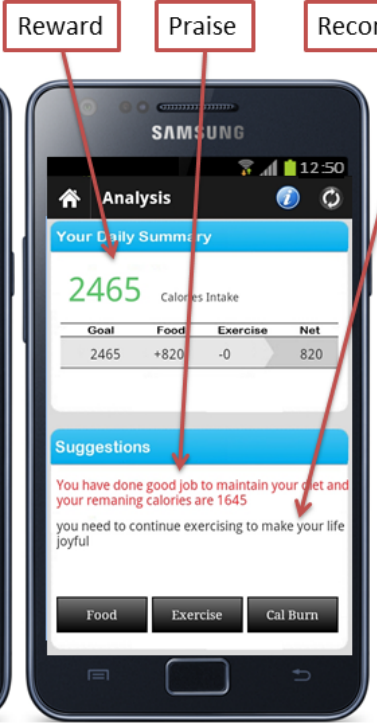

c)

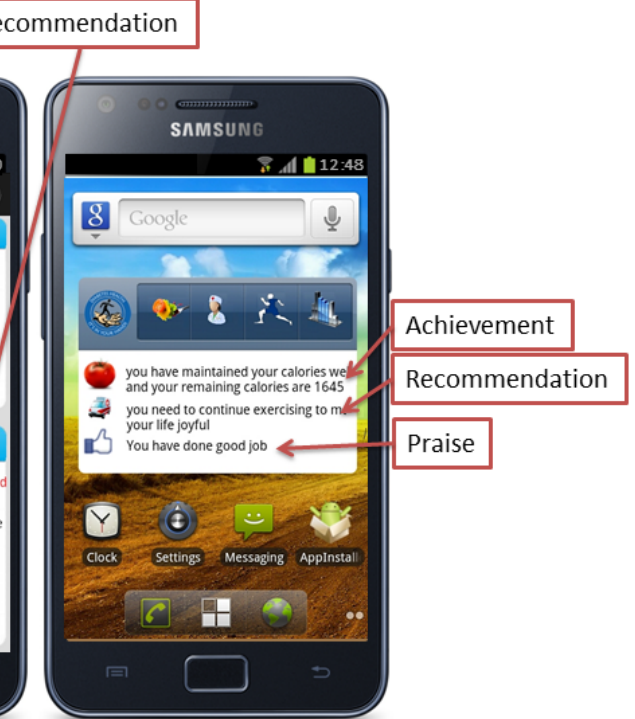

d)

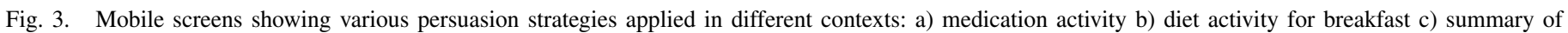

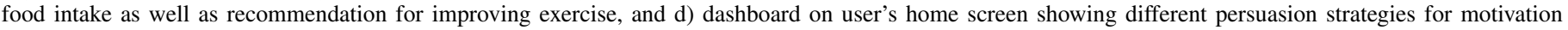
at a glance

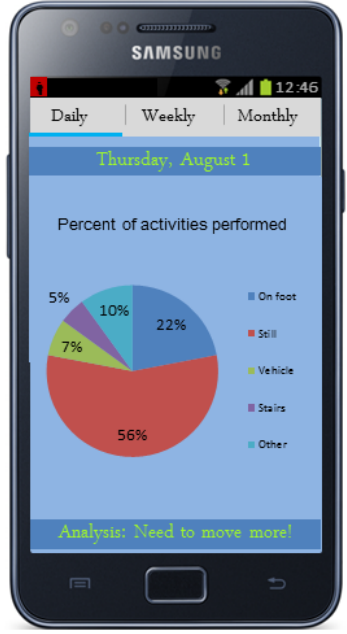

a)

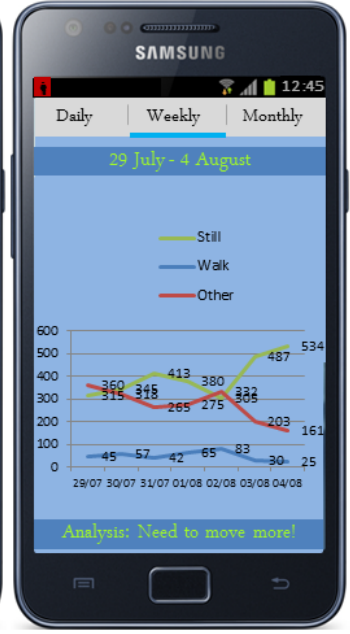

b)
Fig. 4. Mobile views showing user's daily and weekly progress as persuasive graphs to increase user's motivation

updates the user on regular basis about any notifications or events from the social network or experts.

Users record their health-related activities either in their mobile phone where each user maintains a distinct profile. In this prototype implementation, different types of activities entered by users include 1) information about the exercise done, its type, duration, and difficulty level; 2) the medications taken by the user; 3) various parameters related to the type of diet taken including any food taken other than the usual timings. Figure 3 shows different snapshots of our application showing medication and diet-related activities as well as recommendation given to the user based upon these activities. Figure 3 shows the various persuasion strategies used by the application. Figure $3 \mathrm{~d}$ ) also shows the home screen widget containing various updates for the user in the form of different persuasion strategies.

In addition, figure 4 depicts the graphical view of daily and weekly activities as persuasion to the users. High achievers may view these graphs as strengthening while a user performing poorly may see it as a need for improving the physical activities due to the persuasion strategy of "loss aversion."

\section{CONCLUSIONS AND FUtURE WORK}

This paper focuses on the usage of persuasion strategies on smartphones as an application for personalized healthcare management; particularly, we considered the case for diabetic users. Our application combines the activity recognition capabilities of mobile phones with analytics to help users in healthcare management.

This paper briefly described a prototype implementation. Currently, we are enriching our system with existing rules available in different clinical guidelines. Once that is done, we will have a fully deployable system. We will then be able to analyze the usability of our system in a real setup. This will also allow us to evaluate the outcome of various persuasion strategies for better self-management of healthcare. In addition, we are looking for a comparison of different persuasive features in our system with recently developed apps for healthcare.

\section{REFERENCES}

[1] E. Karapanos, "Designing for different stages in behavior change," in Proceedings of the workshop 'Personalization in Persuasive Technology', Persuasive Technology 2016, 2016. 
[2] B. Fogg, "Persuasive technology: using computers to change what we think and do," Ubiquity, vol. 2002, no. December, p. 5, 2002.

[3] H. Mukhtar and D. Belaïd, "Using adaptive feedback for promoting awareness about physical activeness in adults," in In proceedings of the 10th IEEE International Conference on Ubiquitous and Intelligent Computing (UIC) 2013, 2013.

[4] S. Sultan and P. Mohan, "Transforming usage data into a sustainable mobile health solution," Electronic Markets, vol. 23, no. 1, pp. 63-72, 2013.

[5] L. P. Simons and J. F. Hampe, "Exploring e/mhealth potential for health improvement; a design analysis for future e/mhealth impact," $23 \mathrm{rd}$ Bled eConference. from www. bledconference. org, vol. 2, 2010.

[6] H. Kimura, J. Ebisui, Y. Funabashi, A. Yoshii, and T. Nakajima, "idetective: a persuasive application to motivate healthier behavior using smart phone," in Proceedings of the 2011 ACM Symposium on Applied Computing, ser. SAC '11. New York, NY, USA: ACM, 2011, pp. 399404. [Online]. Available: http://doi.acm.org/10.1145/1982185.1982273

[7] I. Albaina, T. Visser, C. van der Mast, and M. Vastenburg, "Flowie: A persuasive virtual coach to motivate elderly individuals to walk," in Pervasive Computing Technologies for Healthcare, 2009. PervasiveHealth 2009. 3rd International Conference on. IEEE, 2009, pp. 1-7.

[8] N. Romero, J. Sturm, T. Bekker, L. De Valk, and S. Kruitwagen, "Playful persuasion to support older adults' social and physical activities," Interacting with Computers, vol. 22, no. 6, pp. 485-495, 2010.

[9] S. Torsi, P. Wright, S. Mawson, G. Mountain, N. Nasr, and B. Rosser, "The self-management of chronic illnesses: Theories and technologies," in Pervasive Computing Technologies for Healthcare (PervasiveHealth), 2010 4th International Conference on-NO PERMISSIONS. IEEE, 2010, pp. 1-4.

[10] S. Ananthanarayan and K. Siek, "Health sense: a gedanken experiment on persuasive wearable technology for health awareness," in Proceedings of the 1st ACM International Health Informatics Symposium. ACM, 2010, pp. 400-404.

[11] V. Parmar, D. Keyson, and C. de Bont, "Persuasive technology to shape social beliefs: A case of persuasive health information systems for rural women in india," Communications of the Association for Information Systems, vol. 24, no. 1, 2009.

[12] S. Consolvo, J. Landay, and D. McDonald, "Designing for behavior change in everyday life," IEEE Computer, vol. 405, pp. 100-103, 2009.

[13] Y. Lin, R. Chen, S. Guo, H. Chang, and H. Chang, "Developing a web 2.0 diabetes care support system with evaluation from care provider perspectives," Journal of Medical Systems, pp. 1-11, 2011.

[14] N. Tatara, E. Arsand, H. Nilsen, and G. Hartvigsen, "A review of mobile terminal-based applications for self-management of patients with diabetes," in eHealth, Telemedicine, and Social Medicine, 2009. eTELEMED'09. International Conference on. IEEE, 2009, pp. 166175.

[15] B. Fogg, "Creating persuasive technologies: An eight-step design process," in Proceedings of the 4th International Conference on Persuasive Technology, April 2009.

[16] I. Ajzen, "The theory of planned behavior," Organizational behavior and human decision processes, vol. 50, no. 2, pp. 179-211, 1991.

[17] B. Fogg and J. Hreha, "Behavior wizard: a method for matching target behaviors with solutions," Persuasive Technology, pp. 117-131, 2010.

[18] B. Fogg, "A behavior model for persuasive design," in Proceedings of the 4th international Conference on Persuasive Technology. ACM, 2009, p. 40.

[19] B. Sheppard, J. Hartwick, and P. Warshaw, "The theory of reasoned action: A meta-analysis of past research with recommendations for modifications and future research," Journal of consumer research, pp. 325-343, 1988.

[20] W. Demark-Wahnefried, E. C. Clipp, I. M. Lipkus, D. Lobach, D. C. Snyder, R. Sloane, B. Peterson, J. M. Macri, C. L. Rock, C. M. McBride et al., "Main outcomes of the fresh start trial: a sequentially tailored, diet and exercise mailed print intervention among breast and prostate cancer survivors," Journal of Clinical Oncology, vol. 25, no. 19, pp. 2709-2718, 2007.

[21] V. B. Stull, D. C. Snyder, and W. Demark-Wahnefried, "Lifestyle interventions in cancer survivors: designing programs that meet the needs of this vulnerable and growing population," The Journal of nutrition, vol. 137, no. 1, pp. 243S-248S, 2007.

[22] A. Khattak, D. Hung, P. Truc, D. Guan, Z. Pervez, M. Han, S. Lee, Y. Lee et al., "Context-aware human activity recognition and decision making," in e-Health Networking Applications and Services (Healthcom), 2010 12th IEEE International Conference on. IEEE, 2010, pp. $112-118$.

[23] A. Khattak, Z. Pervez, K. Ho, S. Lee, and Y. Lee, "Intelligent manipulation of human activities using cloud computing for u-life care," in 2010 10th Annual International Symposium on Applications and the Internet. IEEE, 2010, pp. 141-144.

[24] X. Le, S. Lee, P. Truc, A. Khattak, M. Han, D. Hung et al., "Secured wsn-integrated cloud computing for u-life care," in Consumer Cоттиnications and Networking Conference (CCNC), 2010 7th IEEE. IEEE, 2010, pp. 1-2.

[25] J. García-Vázquez, M. Rodríguez, M. Tentori, D. Saldaña, Á. Andrade, and A. Espinoza, "An agent-based architecture for developing activityaware systems for assisting elderly," Journal of Universal Computer Science (JUCS), vol. 16, no. 12, pp. 1500-1520, 2010.

[26] I. IDF, "Global guidelines for type2 diabetes. available online: http://www.idf.org/webdata/docs/idf\%20ggt2d.pdf, last accessed on 20th mar 2012," 2005.

[27] M. Funnell, T. Brown, B. Childs, L. Haas, G. Hosey, B. Jensen, M. Maryniuk, M. Peyrot, J. Piette, D. Reader et al., "National standards for diabetes self-management education," Diabetes care, vol. 32, no. Supplement 1, p. S87, 2009.

[28] B. Elsawy and K. Higgins, "Physical activity guidelines for older adults," American Family Physician, vol. 81, no. 1, pp. 55-9, 2010.

[29] A. Misra, R. Sharma, S. Gulati, S. Joshi, V. Sharma, A. Ibrahim, S. Joshi, A. Laxmaiah, A. Kurpad, R. Raj et al., "Consensus dietary guidelines for healthy living and prevention of obesity, the metabolic syndrome, diabetes, and related disorders in asian indians," Diabetes Technology \& Therapeutics, vol. 13, no. 6, pp. 683-694, 2011.

[30] T. M. Campbell II, The China study: the most comprehensive study of nutrition ever conducted and the startling implications for diet, weight loss and long-term health. BenBella Books, Inc., 2004.

[31] D. Ornish, The spectrum: a scientifically proven program to feel better, live longer, lose weight, and gain health. Random House Digital, Inc., 2008.

[32] H. Oinas-Kukkonen and M. Harjumaa, "Persuasive systems design: Key issues, process model, and system features," Communications of the Association for Information Systems, vol. 24, no. 1, p. 28, 2009.

[33] J. Whalen, "Persuasive design: Putting it to use," Bulletin of the American Society for Information Science and Technology, vol. 37, no. 6, pp. 16-21, 2011.

[34] R. Cialdini, "The science of persuasion," Scientific American Mind, 2004.

[35] V. Strecher, "Internet methods for delivering behavioral and healthrelated interventions (ehealth)," Аnпи. Rev. Clin. Psychol., vol. 3, pp. 53-76, 2007.

[36] M. Harjumaa, K. Segerståhl, and H. Oinas-Kukkonen, "Understanding persuasive software functionality in practice: a field trial of polar $\mathrm{ft} 60$," in Proceedings of the 4th international Conference on Persuasive Technology. ACM, 2009, p. 2.

[37] P. Chi, J. Chen, H. Chu, and J. Lo, "Enabling calorie-aware cooking in a smart kitchen," Persuasive Technology, pp. 116-127, 2008.

[38] R. C.-S. Chang, H.-P. Lu, P. Yang, and P. Luarn, "Reciprocal reinforcement between wearable activity trackers and social network services in influencing physical activity behaviors," JMIR mHealth and uHealth, vol. 4, no. 3, p. e84, 2016.

[39] HHS, "U.s. department of health and human services. new survey results show huge burden of diabetes," 2009, http://www.nih.gov/news/health/jan2009/niddk-26.htm. 